\title{
Indistinguishability of independent single photons
}

\author{
F. W. Sun* and C. W. Wong \\ Optical Nanostructures Laboratory, Columbia University, New York, NY 10027.
}

(Dated: October 24, 2018)

\begin{abstract}
The indistinguishability of independent single photons is presented by decomposing the single photon pulse into the mixed state of different transform limited pulses. The entanglement between single photons and outer environment or other photons induces the distribution of the center frequencies of those transform limited pulses and makes photons distinguishable. Only the single photons with the same transform limited form are indistinguishable. In details, the indistinguishability of single photons from the solid-state quantum emitter and spontaneous parametric down conversion is examined with two-photon Hong-Ou-Mandel interferometer. Moreover, experimental methods to enhance the indistinguishability are discussed, where the usage of spectral filter is highlighted.
\end{abstract}

PACS numbers: 42.50.Ar, 42.25.Hz, 03.65.Yz

\section{INTRODUCTION}

Linear optical quantum computation [1] is based on the interference between different photons [2], in which the indistinguishability of photons is a fundamental and critical requirement. Any distinguishability will reduce the visibility of interference and the fidelity of quantum computation protocol [3]. It will also directly affect the other applications with photon interference, such as quantum key distribution [4] and high precision quantum phase measurement [5]. Moreover, photon indistinguishability is fundamental to stimulated emission [6, 7] and has been applied in quantum cloning [8, 9] and entanglement measure [10]. Based on the spontaneous parametric down conversion (SPDC), the indistinguishability in the multiphoton interference has been intensely examined recently in experiment [11, 12, 13, 14, 15] and theory $[16,17,18,19]$. However, the kernel is the indistinguishability of independent single photons. In SPDC, independent single photons are heralded by detecting the twinning photons, with several experiments focusing on their indistinguishability and interference [20, 21, 22]. In the solid-state quantum emitters, single photons have been remarkably examined [23, 24, 25, 26, 27, 28], where, in addition to photon statistics and quantum efficiencies, indistinguishability is another important character of the single photon source [22, 28].

Generally, the distinguishability of the single photons comes from the entanglement with extrinsic system, such as photons, phonons or outer environment. Theoretically, the single photons can be described as the mixed state by tracing out the entangling parts. In SPDC, the property of entanglement can be achieved through the analysis of the phase matching condition [22, 29]. However, it is more complicated in the solid-state quantum emitters. Many kinds of physical processes introduce the entanglement between the environment and the emitted single photons. For example, in the single quantum dot, the interaction with phonon results in short dephasing time and gives rises to a very broad spectrum of the photons [30]. This spectrum broadening will make photons

*fs2293@columbia.edu distinguishable. Since it is hard to measure all the physical information of each photon source, the direct analysis of the photon state with interference is highly desired.

In this paper, we will give a brief description of single photons to show the indistinguishability. In the frequency degree of freedom (DOF), the whole photon state is a mixed state of transform limited pulses with different center frequencies. For two independent single photons, there is no entanglement between them. The indistinguishability describes the nature of identicality of the transform limited pulses. To aid in the analysis, we regarded the bandwidth of distribution of these center frequencies as the extrinsic width, which comes form the entanglement between with extrinsic system. The transformed limited pulse are pure state and its width is the intrinsic width. For the same single photon source, single photons have the same extrinsic width and the same intrinsic width. The total spectrum bandwidth is the combination of the intrinsic width and the extrinsic width. Generally, when the extrinsic width is much larger than the intrinsic width, the single photons are totally distinguishable. Only when the extrinsic width is zero, the single photon pulse is transform limited and indistinguishable. In either Lorentzian or Gaussian distributions of the spectrum, the photon indistinguishability is the ratio of intrinsic width to total bandwidth. In experiment, the distinguishability can be measured with Hong-Ou-Mandel (HOM) interferometer [2], where the visibility shows the indistinguishability. In the main section, we will examine the indistinguishability of single photons from solid quantum emitters and SPDC after a general description of the single photon state is given. In the discussion section, experimental methods to enhance the indistinguishability are presented, where the effect of spectral filter is highlighted.

\section{DESCRIPTION AND INDISTINGUISHABILITY OF SINGLE PHOTONS}

We begin the description of single photons from the transform limited pulse, which is a pure quantum state,

$$
|\omega\rangle=\int_{-\infty}^{+\infty} d v g_{\omega}(v) a^{\dagger}(v)|\mathrm{vac}\rangle
$$




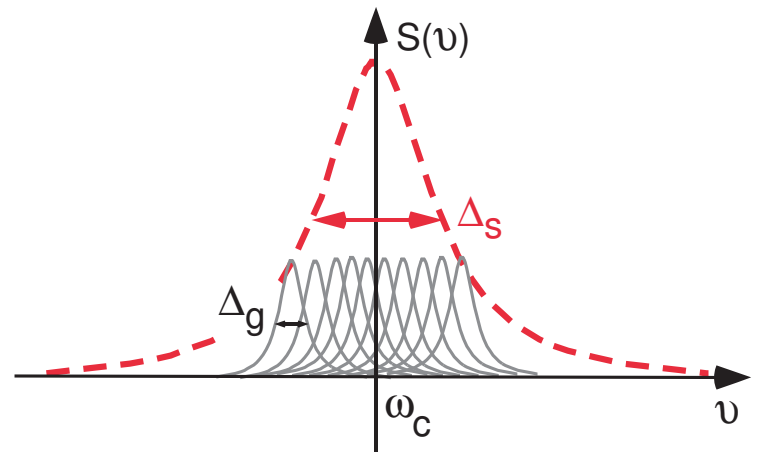

FIG. 1: (color online) Illustration of total single photon pulse (red dashed curve, width $\Delta_{s}$ ) composed of transform limited pulses (grey bold curves, width $\Delta_{g}$ ) with different center frequencies. When $\Delta_{S}=\Delta_{g}$, the single photon pulse is transform limited and indistinguishable.

where $a^{\dagger}(a)$ is the single photon creation (annihilation) operator. $\left|g_{\omega}(v)\right|^{2}$ is the spectrum of the transform limited pulse with center frequency $\omega$ and width $\Delta_{g}$ (intrinsic width). We will discuss the independent single photons from the same source and assume the same $\Delta_{g}$, since the interactions between the single photons and outer environment or other photons are highly similar during the generation. Correspondingly, the transform limited pulse has the duration of $T_{T L}=$ $1 / \Delta_{g}$. Also, $g_{\omega}(v)$ satisfies the normalization condition $\int_{-\infty}^{+\infty} d v\left|g_{\omega}(v)\right|^{2}=1$. The indistinguishability of two independent transform limited photon pulse is $K_{i j}^{T L}=\left|\left\langle\omega_{i} \mid \omega_{j}\right\rangle\right|^{2}$. Roughly, the two photons are totally distinguishable when $\left|\omega_{i}-\omega_{j}\right| \gg \Delta_{g}$ and indistinguishable for $\omega_{i}=\omega_{j}$.

Since the single photons may be entangled with extrinsic system, the center frequencies have the distribution $f(\omega)$ $\left[\int_{-\infty}^{+\infty} d \omega f(\omega)=1\right]$ with width $\Delta_{f}$ (extrinsic width). Then, the whole state is written as

$$
\rho=\int_{-\infty}^{+\infty} d \omega f(\omega)|\omega\rangle\langle\omega|
$$

The total spectrum $S(v)=\int_{-\infty}^{+\infty} d \omega f(\omega)\left|g_{\omega}(v)\right|^{2}$ is broadened to $\Delta_{S} \geq \Delta_{g}$ because of the distribution $f(\omega)$. However, the lifetime of the single photon pulse is same with those transform limited pulses, that is $T_{\rho}=T_{T L}$. Fig. 11 illustrates that the total single photon pulse is composed of different transform limited pulses. Only when $\Delta_{S}=\Delta_{g}=1 / T_{\rho}$ is satisfied, the single photon pulse becomes transform limited.

Formally, the indistinguishability of two independent single photons can be described as

$$
K=\operatorname{tr}(\rho \otimes \rho)=\iint_{-\infty}^{+\infty} d \omega_{i} d \omega_{j} f\left(\omega_{i}\right) f\left(\omega_{j}\right)\left|\left\langle\omega_{i} \mid \omega_{j}\right\rangle\right|^{2} .
$$

If and only if $\rho$ is the pure state, $K=1$. That is with $\Delta_{f}=0$ and $\Delta_{S}=\Delta_{g}$, the single photon states are indistinguishable. On the other hand, when $\Delta_{S} \gg \Delta_{g}, K \rightarrow 0$, the single photon states are distinguishable. From this view, two photons may be distinguishable even when they have the same description. The indistinguishability describes the nature of identicality of the pure state. Eq. (3) also describes the purity of the state $\rho$. Generally, a mixed state comes from an entangled system. In principle, distinguishable information of the state may be obtained by measuring the entangling part. Therefore, the mixed state always has some distinguishability and the purity of the state is a good scale to evaluate the indistinguishability. Moreover, this definition of indistinguishability is highly supported by the experiment. In experiment, the indistinguishable photons will present photon bunching effect and the value of the indistinguishability has the simple relationship with the interference visibility. Based on the single photons from solid-state quantum emitters and SPDC, we will now give detailed discussions on their indistinguishability.

\section{A. Indistinguishability of single photons from single solid quantum emitter}

Here we focus on the single photons from single quantum dot. The single photon from two-level quantum dot spontaneous emission has the Lorentzian distribution,

$$
g_{\omega}(v)=\frac{1}{\sqrt{\pi}} \frac{\sqrt{\Gamma / 2}}{(v-\omega)+i \Gamma / 2},
$$

where $\Gamma / 2$ is the intrinsic width and describes the rate of spontaneous emission [31]. Correspondingly, the lifetime is $T_{1}=1 / \Gamma$. In addition to the intrinsic linewidth, the spectrum broadening mainly comes from the dephasing process. Also, the spectral diffusion of single quantum dot gives much more broader spectrum [32]. All these spectrum broadening can be included in the distribution of $f(\omega)$. For simplicity, we only consider the spectral broadening from pure dephasing which can also be described as the Lorentzian function,

$$
f(\omega)=\frac{1}{\pi} \frac{\Gamma^{\prime}}{\left(\omega-\omega_{c}\right)^{2}+\Gamma^{\prime 2}},
$$

where $\omega_{c}$ is the center frequency of the distribution $f(\omega)$. The extrinsic width is $\Gamma^{\prime}=1 / T_{2}^{\prime}$, where $T_{2}^{\prime}$ is the pure dephasing time. The total state can be described with Eq. (2). The whole spectrum is

$$
S(v)=\frac{1}{\pi} \frac{\Gamma_{2}}{\left(v-\omega_{c}\right)^{2}+\Gamma_{2}^{2}},
$$

where $\Delta_{S}^{L}=\Gamma_{2}=1 / T_{2}=\Gamma^{\prime}+\Gamma / 2$ is the total spectral width and the superscript $L$ in $\Delta_{S}^{L}$ denotes Lorentzian distribution. In the time domain, we get $1 / T_{2}=1 / 2 T_{1}+1 / T_{2}^{\prime}$. When $\Gamma^{\prime}=0, \Gamma_{2}=\Gamma / 2=1 / 2 T_{1}$, the single photons are transform limited.

The indistinguishability of the two transform limited pulses centered at $\omega_{i}$ and $\omega_{j}$ is

$$
K_{i j}^{T L}=\frac{\Gamma^{2}}{\left(\omega_{i}-\omega_{j}\right)^{2}+\Gamma^{2}},
$$

while the indistinguishability of the two single photons with Eq. (3) is

$$
K_{L}=\frac{\Gamma}{2 \Gamma_{2}}
$$


When $\Gamma^{\prime}=0, K_{L}=1$ and the single photon are the transform limited and indistinguishable.

Experimentally, the HOM interferometer is usually used to measure the indistinguishability of two single photons, as shown in Fig 2 a). Two single photons are injected into the two input ports of a 50/50 beamsplitter separately. The twophoton coalescence probability $C_{A B}$ of output ports $A$ and $B$ is null when two photons are indistinguishable and arrive at the beamsplitter simultaneously. Any distinguishability will induce nonzero two photon coalescence probability and reduce the interference visibility. In order to obtain the coalescence probability $C_{A B}(\tau)$ with the interval $\tau$ between the arrival times of two photons, we first calculate probability of two photons exiting in the same output port $C_{A A}(\tau)$, which shows photon bunching when the two photons are indistinguishable [16, 33]. Therefore,

$$
\begin{aligned}
C_{A A}(\tau)= & \frac{1}{8}\left\langle E^{(-)}(t) E^{(-)}(t+\tau) E^{(+)}(t+\tau) E^{(+)}(t)\right\rangle \\
= & \frac{1}{8} \iint_{-\infty}^{+\infty} d \omega_{i} d \omega_{j} f\left(\omega_{i}\right) f\left(\omega_{j}\right) \int_{-\infty}^{+\infty} d t\left\langle\omega_{i}\left|\left\langle\omega_{j}\left|E^{(-)}(t) E^{(-)}(t+\tau) E^{(+)}(t+\tau) E^{(+)}(t)\right| \omega_{j}\right\rangle\right| \omega_{i}\right\rangle \\
= & \frac{1}{4} \iint_{-\infty}^{+\infty} d \omega_{i} d \omega_{j} f\left(\omega_{i}\right) f\left(\omega_{j}\right) \int_{-\infty}^{+\infty} d t\left[\left\langle\omega_{j}\left|E^{(-)}(t) E^{(+)}(t)\right| \omega_{j}\right\rangle\left\langle\omega_{i}\left|E^{(-)}(t+\tau) E^{(+)}(t+\tau)\right| \omega_{i}\right\rangle\right. \\
& \left.+\left\langle\omega_{j}\left|E^{(-)}(t) E^{(+)}(t+\tau)\right| \omega_{i}\right\rangle\left\langle\omega_{i}\left|E^{(-)}(t+\tau) E^{(+)}(t)\right| \omega_{j}\right\rangle\right] \\
= & \frac{1}{4}[1+K(\tau)]
\end{aligned}
$$

where $E^{(+)}(t)=\int_{-\infty}^{+\infty} d \omega a(\omega) e^{-i \omega t} / \sqrt{2 \pi}$ is the detection operator. The coefficient $1 / 8$ comes from photon loss of the beamsplitter $(1 / 4)$ for two photons and the normalization coefficient of two permutations of two photons detecting by two detectors $(1 / 2)$. In the practical experiment, the detection duration is much larger than the photon pulse lifetime and the integral time is extended to $(-\infty,+\infty)$. In the above equation, $K(\tau)$ is the indistinguishability of two photons with time interval $\tau$

$$
\begin{aligned}
K(\tau)= & \iint_{-\infty}^{+\infty} d \omega_{i} d \omega_{j} f\left(\omega_{i}\right) f\left(\omega_{j}\right) \int_{-\infty}^{+\infty} d t \\
& \times\left|\left\langle\omega_{i}\left|E^{(-)}(t) E^{(+)}(t+\tau)\right| \omega_{j}\right\rangle\right|^{2}
\end{aligned}
$$

For the Lorentzian distribution, the indistinguishability is

$$
K_{L}(\tau)=\frac{\Gamma}{2 \Gamma_{2}} e^{-\Gamma|\tau|},
$$

and the two-photon probability is

$$
C_{A A}(\tau)=\frac{1}{4}\left(1+\frac{\Gamma}{2 \Gamma_{2}} e^{-\Gamma|\tau|}\right) .
$$

The excess probability of $K(\tau)$ is the signature of the photon indistinguishability. It is the result of photon bunching from the permutation symmetry of bosonic particles [18, 19].

Because of the symmetry of the beamsplitter, the probability of two photons together in the output port $B$ is same with $C_{A A}(\tau)$. Therefore, the two-photon coincidence probability
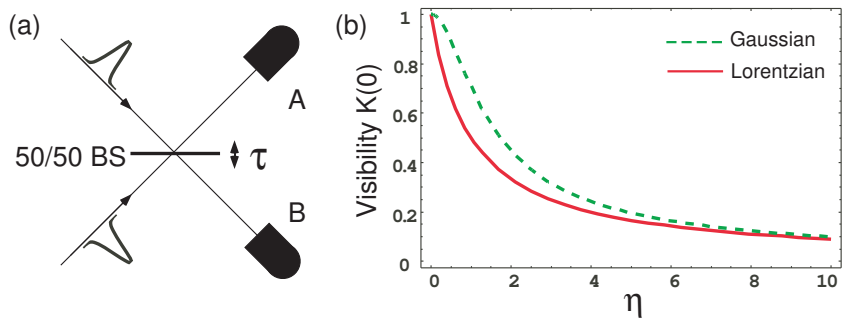

FIG. 2: (color online)(a) Illustration of two-photon Hong-OuMandel interference. $\tau$ is the interval between arrival time of the two input photons. (b) Two-photon Hong-Ou-Mandel interference visibility with different ratios $(\eta)$ of external width to intrinsical width. $\eta=\eta_{L}=2 \Gamma^{\prime} / \Gamma$ for Lorentzian distribution and $\eta=\eta_{G}=\sigma_{f} / \sigma_{g}$ for Gaussian distribution. The visibility approaches $1 / \eta$ when $\eta$ is much larger than 1 .

$C_{A B}(\tau)$ based on the energy conservation law is

$$
\begin{aligned}
C_{A B}(\tau) & =1-2 C_{A A}(\tau) \\
& =[1-K(\tau)] / 2 \\
& =\frac{1}{2}\left(1-\frac{\Gamma}{2 \Gamma_{2}} e^{-\Gamma|\tau|}\right) .
\end{aligned}
$$

$C_{A B}(\tau)$ shows the typical HOM dip with the $1 / e$ width of pulse lifetime, $1 / \Gamma=T_{1}$, as shown in the experimental report [28]. The visibility shows the indistinguishability of $K_{L}$, which is illustrated in Fig. 2(b) with different ratios of extrinsic width to intrinsic width, $\eta=\Delta_{f} / \Delta_{g}$. For Lorentzian distribution, $\eta=\eta_{L}=2 \Gamma^{\prime} / \Gamma$. When extrinsic width is much larger than the intrinsic width, $\eta_{L} \gg 1$, the indistinguishability is approaching to $1 / \eta_{L}$. 


\section{B. Indistinguishability of single photons from SPDC}

In the SPDC, the distinguishability of single photons is induced by the entanglement between the twinning photon. In order to obtain the information of the heralded single photon, the entangling parts need to be traced out in theory. From SPDC, the two-photon state can be written as [29, 34]

$$
|S, I\rangle=\iint_{-\infty}^{+\infty} d \omega_{S} d \omega_{I} \Phi\left(\omega_{S}, \omega_{I}\right) a_{S}^{\dagger}\left(\omega_{S}\right) a_{I}^{\dagger}\left(\omega_{I}\right)|\mathrm{vac}\rangle,
$$

where $\Phi\left(\omega_{S}, \omega_{I}\right)=P\left(\omega_{S}+\omega_{I}\right) H\left(\omega_{S}, \omega_{I}\right)$ is the two-photon wave function, which contains the information of the pump beam spectrum $P\left(\omega_{S}+\omega_{I}\right)$ and the phase matching condition $H\left(\omega_{S}, \omega_{I}\right)$ in the nonlinear crystal. We assume the pump beam is transform limited and the spectrum $\left|P\left(\omega_{S}+\omega_{I}\right)\right|^{2}$ is Gaussian distribution with width $\sigma_{g}$. By making the detection of the idle photon $(I)$ with a single frequency of $\Omega_{I}$, the signal photon $(S)$ has the transform limited single photon state from Eq. (15),

$$
|S\rangle_{\Omega_{2}}=\int_{-\infty}^{+\infty} d \omega_{S} P\left(\omega_{S}+\Omega_{I}\right) H\left(\omega_{S}, \Omega_{I}\right) a_{S}^{\dagger}\left(\omega_{S}\right)|\mathrm{vac}\rangle
$$

Under the normal phase matching condition for thin nonlinear crystal, the bandwidth of $H\left(\omega_{S}, \Omega_{I}\right)$ is much larger than the pump width [35, 36]. Therefore, the $H\left(\omega_{S}, \Omega_{I}\right)$ is slowly varying function and can be taken outside of the integral. In this case, the transform limited single photon pulse has the same shape and width of the pump beam, which can be described with $g_{\omega}(v)=e^{-(v-\omega)^{2} / 4 \sigma_{g}^{2}} / \sqrt[4]{2 \pi \sigma_{g}^{2}}$.

Since the actual detection of the idle photon is the sum of the above detections of different frequency $\Omega_{2}$, the center frequency of the transform limited single photon pulse has the distribution of $f(\omega)$. Without loss of generality, we assume that $f(\omega)=e^{-\left(\omega-\omega_{c}\right)^{2} / 2 \sigma_{f}^{2}} / \sqrt{2 \pi \sigma_{f}^{2}}[35,36]$. Therefore, the heralded single photon can be formally described in Eq. (2) with intrinsic width $\sigma_{g}$ and extrinsic width $\sigma_{f}$. The total spectrum is also Gaussian distribution with the width

$$
\sigma=\sqrt{\sigma_{g}^{2}+\sigma_{f}^{2}}
$$

Moreover, the indistinguishability of two photons with interval $\tau$ is calculated,

$$
K_{G}(\tau)=\frac{\sigma_{g}}{\sigma} e^{-\tau^{2} \sigma_{g}^{2}}
$$

The two-photon coalescence probability $C_{A B}(\tau)$ for the HOM interference is

$$
C_{A B}(\tau)=\frac{1}{2}\left(1-\frac{\sigma_{g}}{\sigma} e^{-\tau^{2} \sigma_{g}^{2}}\right)
$$

with the visibility of $K_{G}(0)=\sigma_{g} / \sigma$, which is also shown in Fig. 2(b) with different ratios of $\eta_{G}=\sigma_{f} / \sigma_{g}$. Moreover, the indistinguishability approaches to $1 / \eta_{G}$ with large extrinsic width. If the two-photon wave function can be factorized, i.e. $\Phi\left(\omega_{S}, \omega_{I}\right)=\Phi\left(\omega_{S}\right) \Phi\left(\omega_{I}\right)$, the single photons is transform limited [22, 29, 34]. In this case, $\sigma_{f}=0$ and the single photons are indistinguishable.

From the results of HOM interference, Eq. (14) and Eq. [19), the width of indistinguishability, or the two-photon fourth-order coherence, only depends on the intrinsic width, or the lifetime of the transform limited pulse. However, the total single photon spectral width determines the width of the single photon second-order coherence time.

\section{DISCUSSION}

\section{A. The definition and the experimental enhancement of indistinguishability}

From the single photon state, the indistinguishability is described in Eq. (3), which is the purity of the state if the single photons are generated in the same source. For the single photons from the different source, the indistinguishability has the description of $K_{i j}=\operatorname{tr}\left(\rho_{i} \otimes \rho_{j}\right)$. At the same time, from the multi-mode theory, the indistinguishability is described as $\mathcal{E} / \mathcal{A}$, where $\mathcal{E}(\mathcal{A})$ is the excess (accidental) two-photon probability [16]. In Refs. [18, 19], the indistinguishability is derived from the coefficients of Schmidt decomposition. All of these definitions are equivalent.

It needs to be emphasized that the extrinsic spectral width comes from the entanglement with extrinsic system. Only this extrinsic spectral width will bring the distinguishability. In the above discussion, we assumed that all other DOFs of the single photon have the same states and no entanglement with the frequency DOF. Actually, the entanglement between the frequency DOF and other inner DOF of the same photon may induce the mixed spectrum description. However, for the same entanglement, the mixed spectrum will not induce the distinguishability when all the DOFs are included, since the entangled state can be described as a linear superposition form for the single photon in a higher dimensional space.

Practically, in order to enhance the indistinguishability, different methods are needed to narrow the extrinsic spectral width or broaden the intrinsic spectral width. For the quantum emitters, low temperature is needed to reduce the interaction with phonons. In this case, the dephasing time is extended [37] and the extrinsic spectral broadening is controlled. Moreover, the interaction with optical cavity mode will decrease the lifetime of the spontaneous emission through Purcell effect [38]. Therefore, the intrinsic width is broadened and the indistinguishability is enhanced [28]. In SPDC, particular design on the phase matching condition helps to generate indistinguishable single photons [22, 29]. However, the usage of spectral filter is the most feasible method to enhance the indistinguishability, especially in the experiment on SPDC.

\section{B. The effect of spectral filter}

In experiment, the narrow spectral filter is widely used to enhance the indistinguishability and interference visibility. 
Theoretically, the detection operator after the spectral filter can be described as

$$
E^{(+)}(t)=\frac{1}{\sqrt{2 \pi}} \int_{-\infty}^{+\infty} d \omega F(\omega) a(\omega) e^{-i \omega t},
$$

where $|F(\omega)|^{2}$ is the spectral transmissivity of the filter. Here we assume the Gaussian distribution of $|F(\omega)|^{2}=$ $e^{-\left(\omega-\omega_{c}\right)^{2} / 2 \sigma_{F}^{2}}$, centered same at $\omega_{c}$ with width $\sigma_{F}$.

With the spectral filter, the spectrum of the single photons is also Gaussian distribution and its width narrows to

$$
\sigma^{\prime}=\frac{\sigma_{F} \sqrt{\sigma_{g}^{2}+\sigma_{f}^{2}}}{\sqrt{\sigma_{g}^{2}+\sigma_{f}^{2}+\sigma_{F}^{2}}} .
$$

At the same time, the filter narrows the intrinsic width,

$$
\sigma_{g}^{\prime}=\frac{\sigma_{F} \sigma_{g}}{\sqrt{\sigma_{g}^{2}+\sigma_{F}^{2}}} .
$$

Using Eq.(18), the indistinguishability is

$$
K_{G}^{\prime}=\frac{\sigma_{g} \sqrt{\sigma_{g}^{2}+\sigma_{f}^{2}+\sigma_{F}^{2}}}{\sqrt{\sigma_{g}^{2}+\sigma_{F}^{2}} \sqrt{\sigma_{g}^{2}+\sigma_{f}^{2}}},
$$

for $\tau=0$.

More rigorously, the effect of the spectral filter extended from Eq.10) is described as:

$$
\begin{aligned}
K_{G}^{\prime}= & \iint_{-\infty}^{+\infty} d \omega_{i} d \omega_{j} f\left(\omega_{i}\right) f\left(\omega_{j}\right) \int_{-\infty}^{+\infty} d t \\
& \times\left|\left\langle\omega_{j}\left|E^{(-)}(t) E^{(+)}(t)\right| \omega_{i}\right\rangle\right|^{2} / C^{2} \\
= & \iint_{-\infty}^{+\infty} d \omega_{i} d \omega_{j} f\left(\omega_{i}\right) f\left(\omega_{j}\right) \\
& \times\left|\int d v F(v) g_{\omega_{i}}(v) F^{*}(v) g_{\omega_{j}}^{*}(v)\right|^{2} / C^{2} \\
= & \frac{\sigma_{g} \sqrt{\sigma_{g}^{2}+\sigma_{f}^{2}+\sigma_{F}^{2}}}{\sqrt{\sigma_{g}^{2}+\sigma_{F}^{2}} \sqrt{\sigma_{g}^{2}+\sigma_{f}^{2}}},
\end{aligned}
$$

where $C$ is the probability to detect the single-photon after the filter

$$
\begin{aligned}
C & =\int_{-\infty}^{+\infty} d \omega f(\omega) \int_{-\infty}^{+\infty} d t\left\langle\omega\left|E^{(-)}(t) E^{(+)}(t)\right| \omega\right)(26) \\
& =\frac{\sigma_{F}}{\sqrt{\sigma_{g}^{2}+\sigma_{f}^{2}+\sigma_{F}^{2}}}
\end{aligned}
$$

Certainly there is photon loss for $C<1$ when using the filter to enhance the indistinguishability.

Fig. 3 shows the effect on the indistinguishability with different ratios of spectral filter width to intrinsic width, $R=$ $\sigma_{F} / \sigma_{g}$. In Fig. 3 a), the width of HOM dip is broadened to
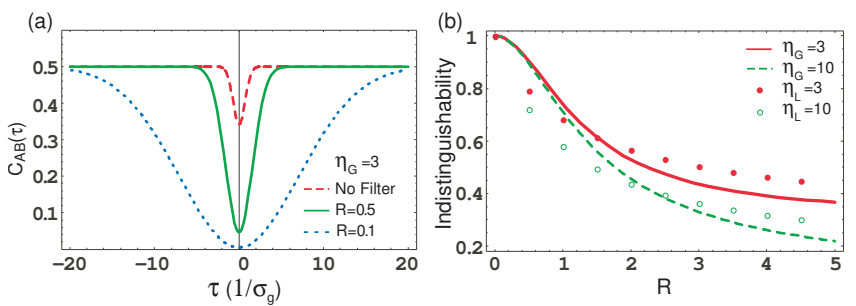

FIG. 3: (color online) Indistinguishability with different widths of filters. (a) Two photons coalescence probability $C_{A B}(\tau)$ of the HOM interference without filter (red dashed curve) and with filters (green solid curve for $R=0.5$ and blue dotted curve for $R=0.1$ ). Here we set $\eta_{G}=3$ for all three cases. The two curves with filters are normalized to the maximal probability of $1 / 2$ for total distinguishable cases $\left(\tau \gg \sigma_{g}^{\prime}\right)$. (b) The red solid and the green dashed curves show the indistinguishability for $\eta_{G}=3$ and $\eta_{G}=10$ with Gaussian filters, respectively. The red solid $\left(\eta_{L}=3\right)$ and green open $\left(\eta_{L}=10\right)$ circles are for the corresponding results of Lorentzian filter on Lorentzian spectrum distribution. In this case, $R=2 \Gamma_{F} / \Gamma$, where $\Gamma_{F}$ is the Lorenzian filter width.

$1 / \sigma_{g}^{\prime}$, since the intrinsic width is narrowed by the spectral filter in Eq.(22). Fig. 3 b) shows the indistinguishability with $\eta_{G}=3$ (red solid) and $\eta_{G}=10$ (green dashed). In comparison, the results of the Lorentzian filter on Lorentzian spectrum distribution are also shown in Fig. 3 (b) with red solid $\left(\eta_{L}=3\right)$ and green open $\left(\eta_{L}=10\right)$ circles. These results are numerically calculated with Eq.(24) and Eq.(26). Clearly, the indistinguishability is approaching to 1 when the filter width is closing to 0 . For $\eta \gg 1$, the value of the indistinguishability shows the same result as in Fig. 2(b), where the extrinsic width is replaced by the filter width.

In SPDC, for the pump pulse duration of 110fs (full width at half maximum), the indistinguishability with a full width at half maximum $3 \mathrm{~nm}$ filter is about 0.94 for $\eta \gtrsim 3$ [36]. It is little higher than the experimental results in [5, 14] because there may be entanglement in other degrees of freedom between the twin photons besides the frequency entanglement [18]. Here, we used the condition that the single photon intrinsic width $\sigma_{g}$ is same with the pump beam width for thin nonlinear crystal.

\section{Independent photons from many quantum emitters}

In some cases, there is more than one independent photon from many quantum emitters. The total state is

$$
\rho_{N}=\prod_{i=1}^{N} \rho_{k} .
$$

where $\rho_{k}=\left(C|\mathrm{vac}\rangle\left\langle\mathrm{vac}\left|+\int_{-\infty}^{+\infty} d \omega f_{k}(\omega)\right| \omega\right\rangle\langle\omega|\right)$ is for the independent single photon with $C=1-\int_{-\infty}^{+\infty} d \omega f_{k}(\omega)$. Considering the photon loss in the practical experiment and quantum efficiency of the quantum emitters, $\int_{-\infty}^{+\infty} d \omega f_{k}(\omega)<$ 1. Moreover, $f_{k}(\omega)$ may have different center wavelengthes. For example, there is size distribution of quantum dots. In this 
case, the total spectrum will include the broadening from size distribution. Therefore, the spectrum is very broad and the photons will be distinguishable even at the low temperature.

\section{CONCLUSION}

The description of the single photons state in the spectrum domain is presented to discuss the indistinguishability. The ratio of extrinsic spectrum width to intrinsic width governs the indistinguishability. Single photons are indistinguishable only when they have the same transform limited forms, while they are highly distinguishable when the extrinsic spectrum width is much larger than the intrinsic width. Fundamentally, the indistinguishability of independent photons shows the sameness of part which can be described with pure state and only the indistinguishable parts can interfere each other. In experiment, the indistinguishability shows excess probability of two-photon coincident detection in Hanbury-Brown-Twiss interferometer [33] or less probability in HOM interferometer. Moreover, the indistinguishability can be experimentally enhanced with the narrow spectral filter or by controlling the generation condition.

\section{Acknowledgments}

F.W.S. thanks Z. Y. Ou for helpful discussion. This work is funded by DARPA, NSF Contract No. ECCS 0747787, and the New York State Office of Science, Technology and Academic Research.
[1] P. Kok, et al., Rev. Mod. Phys. 79, 135 (2007).

[2] C. K. Hong, Z. Y. Ou, and L. Mandel, Phys. Rev. Lett. 59, 2044 (1987).

[3] P. P. Rohde and T. C. Ralph, Phys. Rev. A 71, 032320 (2005).

[4] N. Gisin, G. Ribordy, W. Tittel, and H. Zbinden, Rev. Mod. Phys. 74, 145 (2002).

[5] F. W. Sun, B. H. Liu, Y. X. Gong, Y. F. Huang, Z. Y. Ou, and G. C. Guo, Europhys. Lett. 82, 24001 (2008).

[6] A. Lamas-Linares, J. C. Howell, and D. Bouwmeester, Nature 412, 887 (2001).

[7] F. W. Sun, B. H. Liu, Y. X. Gong, Y. F. Huang, Z. Y. Ou, and G. C. Guo, Phys. Rev. Lett. 99, 043601 (2007).

[8] A. Lamas-Linares, C. Simon, J. C. Howell, and D. Bouwmeester, Science 296, 712 (2002).

[9] S. Fasel, N. Gisin, G. Ribordy, V. Scarani, and H. Zbinden, Phys. Rev. Lett. 89, 107901 (2002).

[10] F. W. Sun, et al., Phys. Rev. A 76, 052303 (2007).

[11] Z. Y. Ou, J. -K. Rhee, and L. J. Wang, Phys. Rev. Lett. 83, 959 (1999).

[12] K. Tsujino, H. F. Hofmann, S. Takeuchi, and K. Sasaki, Phys. Rev. Lett. 92, 153602 (2004).

[13] H. S. Eisenberg, J. F. Hodelin, G. Khoury, and D. Bouwmeester, Phys. Rev. Lett. 96, 160404 (2006).

[14] G. Y. Xiang, Y. F. Huang, F. W. Sun, P. Zhang, Z. Y. Ou, and G. C. Guo, Phys. Rev. Lett. 97, 023604 (2006).

[15] B. H. Liu, F. W. Sun, Y. X. Gong, Y. F. Huang, Z. Y. Ou, and G. C. Guo, Europhys. Lett. 77, 24003 (2007).

[16] Z. Y. Ou, J. -K. Rhee, and L. J. Wang, Phys. Rev. A 60, 593 (1999).

[17] Z. Y. Ou, Phys. Rev. A 72, 053814 (2005); ibid, 74, 063808 (2006); ibid, 77, 043829 (2008).

[18] F. W. Sun, B. H. Liu, Y. F. Huang, Y. S. Zhang, Z. Y. Ou, and G. C. Guo, Phys. Rev. A 76, 063805 (2007).

[19] F. W. Sun, B. H. Liu, C. W. Wong, and G. C. Guo, Phys. Rev. A, 78, 015804 (2008).

[20] H. de Riedmatten, I. Marcikic, W. Tittel, H. Zbinden, and N.
Gisin, Phys. Rev. A 67, 022301 (2003).

[21] R. Kaltenbaek, B. Blauensteiner, M. Żukowski, M. Aspelmeyer, and A. Zeilinger, Phys. Rev. Lett. 96, 240502 (2006).

[22] P. J. Mosley et al., Phys. Rev. Lett. 100, 133601 (2008).

[23] F. De Martini, G. Di Giuseppe, and M. Marrocco, Phys. Rev. Lett. 76, 900 (1996).

[24] J. Kim, O. Benson, H. Kan, and Y. Yamamoto, Nature 397, 500 (1999).

[25] P. Michler, et al., Science 290, 2282 (2000).

[26] C. Santori, M. Pelton, G. Solomon, Y. Dale, and Y. Yamamoto, Phys. Rev. Lett. 86, 1502 (2001).

[27] A. Kuhn, M. Hennrich, and G. Rempe, Phys. Rev. Lett. 89, 067901 (2002).

[28] C. Santori, D. Fattal, J. Vučković, G. S. Solomon, and Y. Yamamoto, Nature 419, 594 (2002).

[29] A. B. U'Ren et al., Laser Phys. 15, 146 (2005); A. B. U'Ren, Y. Jeronimo-Moreno, and H. Garcia-Gracia, Phys. Rev. A 75, 023810 (2007).

[30] H. J. Carmichael, Statistical methods in quantum optics: master equations and fokker-planck equations (Springer, 1999); P. Borri and W. Langbein, in Single Quantum Dots: Fundamentals, Application and New Concepts, P. Michler, Ed., vol. 90 of Springer Series in Topics in Applied Physics (Springer, 2003).

[31] M. O. Scully and M. S. Zubairy, Quantum optics (Cambridge University Press 1997).

[32] R. G. Neuhauser, K. T. Shimizu, W. K. Woo, S. A. Empedocles, and M. G. Bawendi, Phys. Rev. Lett. 85, 3301 (2000).

[33] R. Hanbury Brown and R. Q. Twiss, Nature 177, 27 (1956).

[34] Z. Y. Ou, Quantum Semiclassic. Opt. 9, 599 (1997); Multiphoton quantum interference (Springer, 2007).

[35] W. P. Grice and I. A. Walmsley, Phys. Rev. A 56, 1627 (1997).

[36] Y. H. Kim and W. P. Grice, Opt. Lett. 30, 908 (2005).

[37] P. Borri, et al., Phys. Rev. Lett. 87, 157401 (2001).

[38] E. M. Purcell, Phys. Rev., 69, 37 (1946). 